\title{
EL CAFÉ Y SUS EFECTOS EN LA SALUD CARDIOVASCULAR Y EN LA SALUD MATERNA
}

\section{COFFEE, CAFFEINE, AND ITS EFFECTS ON CARDIOVASCULAR HEALTH AND MATERNAL HEALTH}

\author{
Alfonso Valenzuela B. \\ Centro de Lípidos, INTA, Universidad de Chile y Facultad de Medicina, \\ Universidad de los Andes. Santiago, Chile.
}

\begin{abstract}
Caffeine is the principal active component of coffee and the effects of coffee consumption are mainly associated to this component. Caffeine is a methylxanthine which acts as antagonist of adenine receptors at the nervous system. A great number of health effects, both beneficial and detrimental, have been associated to caffeine consumption: such as cardiovascular disease, type 2 diabetes, glucose tolerance and insulin sensitivity, hepatic cirrhosis, hepatocellular carcinoma, are included as the main targets. The present work reviews two of the main concerns about coffee and caffeine consumption: cardiovascular disease and maternal health (perinatal period). The general conclusion is that caffeine consumption up to $300 \mathrm{mg} /$ day does not constitute a risk of myocardial infarction, hypertension, or modification of cardiovascular risk markers such as reactive $C$ protein or homocysteine plasmatic levels. Similar conclusions are reached about the maternal health. Moderate caffeine consumption (300 mg/day or less) does not constitute a major risk of less conception, increased gestational diabetes, decrease fetal growth, or congenital defects. However, absence of risk may not be interpreted as a signal to stimulate coffee consumption during pregnancy. Future mothers, which are moderated coffee consumers, may be suggested to continue this consumption during pregnancy, but under medical supervision.
\end{abstract}

Key words: Coffee consumption, caffeine ingestion, metabolic effects, cardiovascular health, maternal health.

Este trabajo fue recibido el 23 de Marzo de 2010 y aceptado para ser publicado el 25 de Septiembre de 2010.

\section{INTRODUCCIÓN}

Después de los aceites comestibles y el té, el café es el producto del tipo "commodity" más consumido en el mundo (1). Se estima que al menos el $30 \%$ de la población mundial consume una vez al día una taza de café. Entre de los grandes consumidores están los europeos, especialmente los países nórdicos, los norteamericanos, y los países latinoamericanos tradicionalmente productores de café como Colombia y Brasil. El atractivo del café esta ligado a su aroma, sabor, al placer de degustar una "taza de café" consumida en solitario o con compañía, y al concepto generalizado que el café es "estimulante" y "energizante". Habitualmente el consumo de café se asocia al tabaquismo, hábito que en muchos estudios resulta confundente, debido a los efectos negativos demostrados en el hábito de fumar. Las actividades laborales y profesionales que demandan un mayor estrés, siempre se asocian a un mayor consumo de café. ¿Qué contiene el café que lo hace tan atractivo de consumir?, ¿es beneficioso su consumo?

El café, preparado ya sea por ebullición y posterior decantación (café turco o griego) o por filtración del extracto de ebullición por simple gravedad (usando papel filtro), o mediante presión (café expresso), contiene una serie de sustancias que han sido motivo de estudio durante muchos años (2). Sin lugar a dudas, la cafeína es la más importante, pero también el café aporta sustancias como el cafestol, el kahweol, el ácido clorogénico a los cuales se les atribuye propiedades antioxidantes (3). Además, contiene una serie de micronutrientes como el magnesio, potasio, niacina, trigenolina, tocoferoles, entre otros a los cuales se les ha atribuido, con mayor o menor impacto, efectos benéficos (3). Son numerosos los efectos en la salud atribuidos al consumo de café y particularmente 
a la cafeína, efectos tanto benéficos como deletéreos, lo cual tiende a confundir, principalmente debido a la abundante información disponible en ambos sentidos.

El consumo de café se ha relacionado con la salud cardiovascular, la diabetes tipo 2 , la tolerancia a la glucosa y la sensibilidad a la insulina, el daño hepático debido a cirrosis y el carcinoma hepatocelular, entre otros. También se ha relacionado el consumo de cafeína con el período maternal, así como en la salud de la tercera edad. Debido a la abundancia de información, esta revisión solo se centrará, en forma no exhaustiva, en dos aspectos relacionados con el consumo de cafeína: sus efectos en la salud cardiovascular y durante el período maternal (gestacional y post natal).

\section{LA CAFEINA, EL PRINCIPAL PRINCIPIO ACTIVO DEL CAFÉ}

La cafeína (1,3,7-metilxantina) es un alcaloide de estructura purínica que se encuentra naturalmente en los granos del café. El 75\% del consumo de cafeína mundial es aportado por el café, un $15 \%$ por el consumo de té y un $10 \%$ por las bebidas energéticas adicionadas de cafeína (4). Independiente del proceso de preparación del café para su consumo (hervido o filtrado), en promedio una taza de café $(150 \mathrm{cc})$ aporta entre 90 y 200 mg de cafeína, dependiendo eso sí del origen del café, de la modalidad de preparación, e incluso del lugar (local) donde se prepara (5). Curiosamente, un estudio demostró que el café expendido por un mismo local en seis días seguidos varió en su contenido de cafeína de $132 \mathrm{mg}$ a $282 \mathrm{mg}(3)$.

La cafeína se absorbe casi totalmente en el estómago y en el intestino delgado, distribuyéndose en casi todos los tejidos, incluido el cerebro ya que el alcaloide es muy permeable a la selectiva barrera hémato-encefálica. La metabolización de la cafeína ocurre primariamente en el hígado (95\%), tejido en el que una isoforma del citocromo P-450 (CYP1A2) demetila la cafeína a 1,7dimetilxantina (paraxantina), la que posteriormente es nuevamente demetilada y transformada por oxidación en ácido 1-metilúrico, el cual puede ser eliminado a través de la orina (6). Existen otras vías alternativas de metabolización de menor importancia (3). La velocidad de metabolización de la cafeína es un determinante importante en el análisis de sus efectos metabólicos. Es así como individuos homocigotos para la expresión del CYP1A2, identificado como subclase *1A, son metabolizadores rápidos de la cafeína y corresponden al $45 \%$ de la población caucásica. En cambio, aquellos que poseen la subclase $* 1 \mathrm{~B}$ se identifican como metabolizadores lentos de la cafeína y corresponden aproximadamente al 55\% de la población caucásica (7).
Metabólicamente, la cafeína es un antagonista competitivo de los receptores de adenosina A1 y del subtipo A2a (8). Los receptores de adenosina se asocian a las proteínas $\mathrm{G}$ y se encuentran en alta concentración en diferentes áreas del cerebro, incluyendo el hipocampo y el núcleo talámico (9). La adenosina es un neuromodulador que produce efectos inhibitorios, tanto en el sistema nervioso central como periférico, por lo cual el antagonismo que ejerce la cafeína sobre este modulador produce, generalmente, efectos estimulantes (10). Adicionalmente, se ha identificado que la cafeína inhibe receptores de GABA, cual es el principal neurotransmisor inhibitorio cerebral (11). La figura 1 muestra la estructura de la cafeína y de la adenosina.

\section{LA CAFEINA Y SUS EFECTOS EN LA SALUD}

Se han realizado numerosos estudios epidemiológicos en diferentes países destinados a caracterizar los efectos bioquímicos y fisiológicos de la cafeína y así poder diferenciarlos de otras variables potencialmente confundentes, como son el tabaquismo y el consumo de alcohol. Generalmente, el consumo de café se estima a partir de encuestas de consumo de alimentos, pero el tamaño de la "tacita" o "taza" de café puede ser muy variable, desde $50 \mathrm{cc}$ hasta $250 \mathrm{cc}$, lo cual complica la comparación entre los diferentes estudios. Sin embargo, una revisión crítica permite obtener conclusiones estadísticamente válidas a partir de varios estudios sobre el posible efecto del café, y de la cafeína, en las enfermedades cardiovasculares y durante el período gestacional y post natal. Algunos estudios están referidos específicamente al efecto de la cafeína, en tanto que otros relacionan el consumo de café, lo cual implica no sólo la presencia de cafeína, sino de numerosos otros compuestos que contiene el café, algunos de ellos ya mencionados, que también tienen efectos en la salud, principalmente antioxidantes.

\section{LA CAFEINA Y LAS ENFERMEDADES CARDIOVASCULARES}

Son numerosos los estudios epidemiológicos que han examinado la relación entre el consumo de café (o cafeína) y el mayor o menor riesgo de enfermedad cardiovascular. El análisis de los resultados puede resultar confundente, ya que en algunos estudios la conclusión es que el consumo de café constituye un riesgo para la salud cardiovascular (12), en tanto que otros concluyen que, no tiene efectos (13), incluso que es beneficioso (14). A continuación se resumen los principales resultados sobre el efecto del café y/o de la cafeína en la enfermedad cardiovascular y en los factores de riesgo que determinan esta compleja patología. 


\section{Enfermedad coronaria e infarto al miocardio (IM).}

En general, casi todos los estudios epidemiológicos, prospectivos y de meta-análisis, concluyen que un consumo alto de café puede constituir un mayor riesgo de enfermedad cardiovascular (3-12-13-15). Sin embargo, un análisis en detalle de estos estudios, lleva a concluir que el riesgo, particularmente de IM, presenta una curva tipo J (16), característica en muchos estudios epidemiológicos. Esto es, el no consumo de café se asocia con un riesgo menor a moderado de IM, el consumo de cantidades moderadas de café origina un bajo riesgo de IM y cantidades crecientes de consumo implican riesgos mayores de IM. La figura 2 grafica las diferencias en el riesgo de IM o de mortalidad por enfermedad coronaria y el consumo de café. De esta figura se puede apreciar que el consumo de $300 \mathrm{~mL} /$ día o menos de café, representa un factor de protección al IM, incluso superior al no consumo de café. Sobre $600 \mathrm{~mL} /$ día el riesgo comienza a incrementarse. Es probable que la protección observada en el rango de consumo hasta $300 \mathrm{~mL} /$ día sea, además, una consecuencia del efecto protector de los polifenoles antioxidantes que contiene el café (cafestol, kahweol, ácido clorogénico, ácido ferúlico, ácido cumárico, entre otros), más que a un efecto específico de la cafeína (14). $\mathrm{El}$ ascenso de la curva representaría el efecto negativo de la cafeína, el que en este rango sería superior al efecto protector de los antioxidantes contenidos en el café (14).

El efecto del café y de la cafeína en pacientes con enfermedad cardiovascular ya establecida ha sido poco estudiado. Un solo estudio caso-control concluye que el consumo de 10 o más tazas de café produce un aumento significativo de IM en pacientes con la patología ya establecida (17). Sin embargo, un estudio multicéntrico en pacientes sobrevivientes de un IM no encontró asociación entre el consumo de café y la supervivencia hasta cuatro años después del IM, aún en aquellos con alto consumo de café (18).

\section{Cafeína e hipertensión}

La hipertensión es uno de los mayores riesgos de enfermedad cardiovascular, por lo cual aún pequeñas reducciones en la presión vascular pueden tener un gran impacto en la salud pública (19). La cafeína puede aumentar los niveles plasmáticos de hormonas relacionadas al estrés, como la adrenalina, noradrenalina y el cortisol, por lo cual podría esperarse un efecto hipertensivo derivado del consumo de café, ya que la secreción de estas hormonas es estimulada por la cafeína (20). Sin embargo, del Estudio de la Enfermeras en sus etapas I y II no se deduce una relación entre el consumo de cafeína y la hipertensión (21). Por otro lado, las bebidas energizantes

\section{FIGURA 1}

\section{Estructura química de cafeín y adenosina}
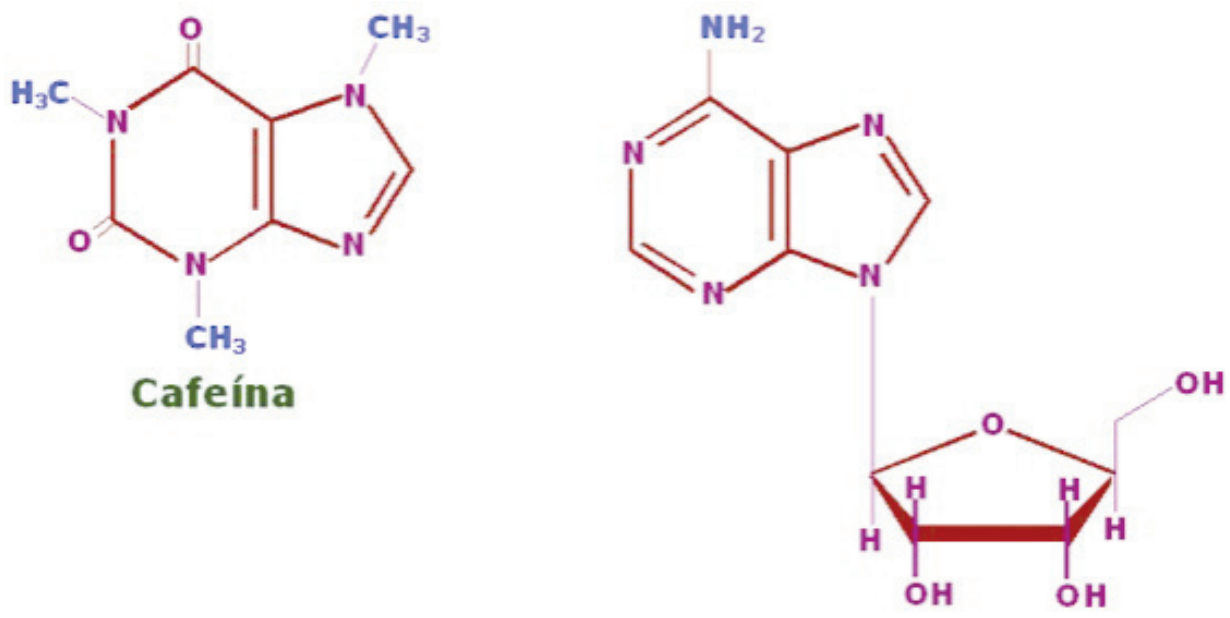

Adenosina 
que contienen cafeína, sí producen hipertensión (22). En base a esta información, los investigadores concluyen que los antioxidantes contenidos en el café y que no contienen las bebidas energizantes, contrarrestan el posible efecto hipertensor observado pata la cafeína (23).

\section{Arritmias cardíacas y el consumo de café}

Las arritmias cardíacas, ventriculares y atrioventriculares, se producen por daño estructural del músculo cardíaco debido a aterosclerosis, hipertensión, enfermedades valvulares, y cardiomiopatías, entre otras causas, y son complicaciones frecuentes derivadas de un IM. En un extenso estudio realizado en Dinamarca, país que se caracteriza por su alto consumo de café y derivado del Estudio de Dieta, Cáncer y Salud Danés, que incluyó 48000 sujetos, se concluye que el consumo de hasta diez tazas de café al día, equivalente 997 mg de cafeína, no tiene mayor incidencia en el riesgo de arritmias cardíacas (24).

\section{Inflamación y función endotelial}

Los niveles plasmáticos aumentados de la proteína $C$ reactiva, un activador de los monocitos en el endotelio vascular, son considerados marcadores moleculares de procesos inflamatorios y un factor de riesgo de enfermedad cardiovascular. El Estudio de las Enfermeras no demostró que el consumo de café altera los niveles de la proteína $\mathrm{C}$ reactiva en las mujeres sanas. Más aún, en mujeres diabéticas los niveles de este marcador fueron disminuidos por un consumo de moderado a alto de café (25). La conclusión general es que el consumo moderado de café no afecta en forma significativa los niveles plasmáticos de la proteína $\mathrm{C}$ reactiva y de otros marcadores inflamatorios (14).

\section{Cafeína y homocisteína}

Los niveles plasmáticos de la homocisteína se asocian con un incremento en el riesgo de enfermedad cardiovascular, ya que los altos niveles de este sulfoaminoácido se correlacionan con un mayor riesgo de IM. La homocisteína es un producto del metabolismo de la metionina y sus niveles plasmáticos aumentan cuando hay deficiencia de folato, vitamina B6 y vitamina B12. El consumo de cafeína se ha asociado de una manera dosis-dependiente con mayores niveles plasmáticos de homocisteína (26), del mismo modo que la abstención

\section{FIGURA 2}

Efecto del consumo de café sobre la mortalidad por enfermedades cardiovasculares.

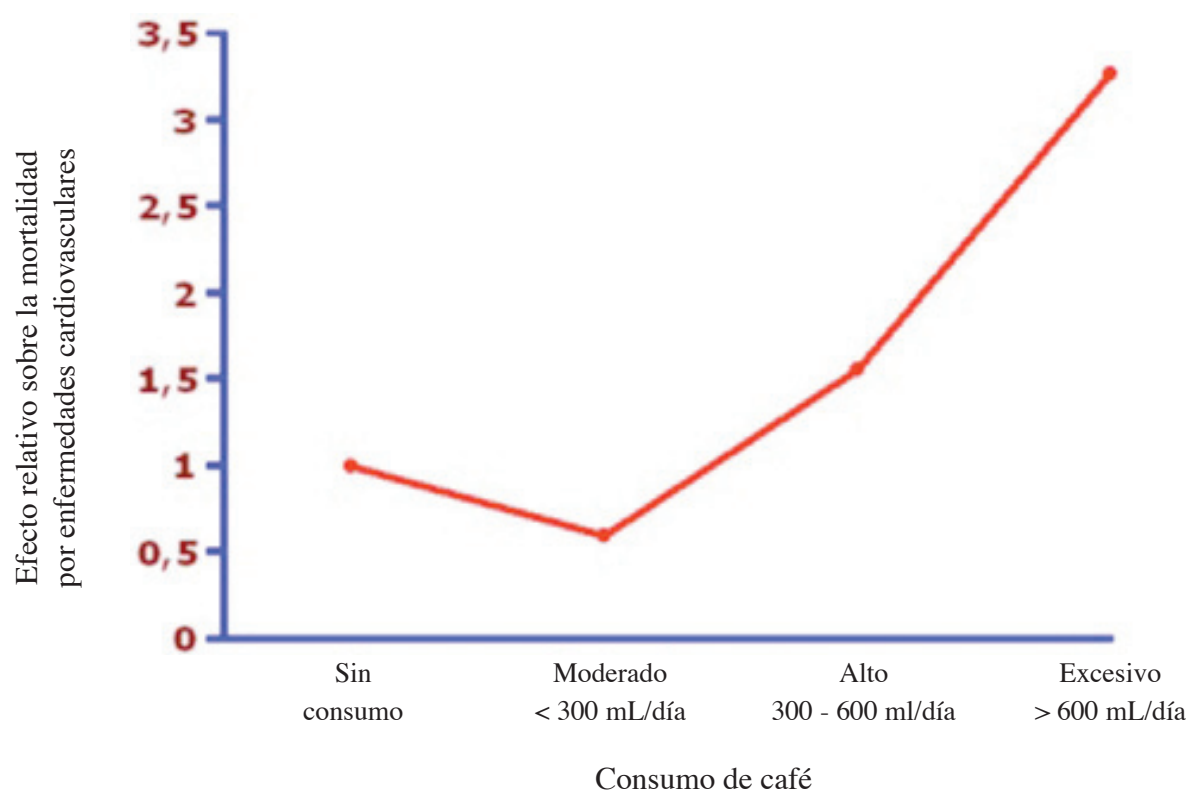


del consumo de café produce reducciones de hasta un $11 \%$ en los niveles del marcador molecular (27). Existe una relación muy directa entre el consumo de ácido fólico y los niveles plasmáticos de homocisteína, ya que una adecuada nutrición o suplementación con ácido fólico constituye un factor de protección a la formación de altos niveles de homocisteína (28). Se ha observado que el efecto de la cafeína en los niveles de homocisteína es más marcado en aquellos individuos homocigotos para la enzima metionina-tetrahidrofolato reductasa, importante en la formación de ácido fólico (28). De esta forma, se ha propuesto que solo los individuos que presentan deficiencias de ácido fólico verían afectados sus niveles plasmáticos de homocisteína por el consumo de cafeína (29).

En un trabajo reciente López-García et al (30), en el cual se realizó un elaborado estudio de dos cohortes (41.736 hombres y 86.214 mujeres) derivadas del Estudio de los Profesionales de la Salud y del Estudio de las Enfermeras, seleccionando individuos sin antecedentes de enfermedad cardiovascular, se concluyó que el consumo regular de café no se asocia a un incremento del riesgo de mortalidad por enfermedad cardiovascular, tanto en hombres como en mujeres. Incluso, los investigadores también asocian el consumo de café a un bajo riesgo desarrollo de cáncer. Los resultados de este trabajo complementan los obtenidos por el mismo grupo en 2006 (31), reafirmando la no vinculación entre el consumo moderado de café y el mayor riesgo de enfermedad cardiovascular. Más recientemente, el grupo de investigadores integrantes del Amsterdam Growth and Health Longitudinal Study (AGAHLS) concluye que el consumo moderado de café no se asocia con ninguno de los componentes del síndrome metabólico, incluida la ausencia de efecto sobre la presión arterial (32).

\section{EL CAFÉ Y LA CAFEINA EN EL EMBARAZO Y LA SALUD PERINATAL}

\section{Metabolismo de la cafeína en la placenta, el feto y el recién nacido}

La cafeína puede atravesar libremente la placenta con lo cual se estima que la concentración de cafeína en el líquido amniótico y en la sangre materna constituyen indicadores de la concentración de cafeína en la sangre fetal (33). Ni el feto ni la placenta tienen la capacidad para metabolizar la cafeína, con lo cual se estima que si la madre toma café, el feto estará continuamente expuesto a la cafeína que deriva de la sangre materna a la placenta, tejido que no puede metabolizar la cafeína debido a que solo cuenta con el CYP1A1 y no con el CYP1A2, la isoenzima del citocromo P-450 que metaboliza a la cafeína (34). Hasta los ocho meses de gestación, el feto no posee las enzimas hepáticas necesarias para la metabolización de la cafeína (35). El recién nacido es también incapaz de metabolizar totalmente la cafeína, por lo cual como mecanismo compensatorio la elimina como tal en la orina (90\%), a diferencia del adulto que sólo elimina $2 \%$ de la cafeína siendo el resto metabolizada (36).

La vida media de la cafeína en el neonato se estima en el rango de 50 a 100 horas, siendo solo de 6 horas en el adulto. Sin embargo, ya a las pocas semanas de vida extrauterina el tiempo de metabolización va disminuyendo paulatinamente siendo este similar al del adulto a las doce semanas (37). Debido a que la cafeína inhibe a la enzima fosfodiesterasa hepática y muscular, con lo cual produce una menor metabolización del AMPc (38), se ha propuesto que la metilxantina podría causar vasoconstricción tanto en el útero materno como en el feto e incluso en el recién nacido, aunque este aspecto no ha sido comprobado experimentalmente (21). Si efectivamente la cafeína produce vasoconstricción uterina y fetal podría, entonces, disminuir la irrigación sanguínea y la oxigenación del tejido placentario y del feto, lo cual podría producir alteraciones en el crecimiento y la nutrición fetal (39). Sin embargo, los antecedentes clínicos y epidemiológicos que se discuten más adelante, parecen no validar estas posibles anomalías derivadas del consumo de café y cafeína.

El consumo de café por parte de las mujeres es relativamente alto en algunos países. En Estados Unidos $25 \%$ de las mujeres entre 20 y 29 años consume café habitualmente, el consumo aumenta al $46 \%$ de las mujeres entre 30 y 39 años (40). Considerando que estos rangos de edad corresponden al período fértil de la mujer, numerosos investigadores han estudiado la asociación entre el consumo de café y cafeína durante el embarazo y el período perinatal.

\section{El consumo de cafeína y la concepción}

Numerosos estudios han examinado la relación entre el consumo de cafeína y el tiempo de concepción en mujeres fértiles (41). La mayoría no ha encontrado asociación entre la cafeína o el consumo de café y una menor fertilidad o una mayor duración de la fecundación (42-43). Algunos estudios han evidenciado que sólo el consumo de cantidades altas de cafeína (400-600 mg/día) podría correlacionarse con un mayor tiempo para lograr la fecundación (43). Sin embargo, estos estudios han sido criticados debido a que no tienen controles adecuados en relación al estilo de vida de las mujeres investigadas, particularmente en relación al tabaquismo y el consumo de alcohol (44). La conclusión general es que consumos de cafeína hasta $300 \mathrm{mg} /$ día no constituirían un riesgo 
de atrasar la concepción durante los períodos fértiles de la mujer (3).

\section{La cafeína y las complicaciones del embarazo}

Siendo el aborto espontáneo una de las complicaciones mas graves del embarazo, esta patología ha sido motivo de numerosos estudios relacionados con el posible efecto de la cafeína. La metodología de los estudios ha sido dispar, principalmente en la estimación real del consumo de café de las embarazadas y en la identificación de factores confundentes como las nauseas, que podría llevar al no consumo de café, y el consumo de alcohol (45). Sin embargo, la conclusión general es que consumos no superiores a $300 \mathrm{mg} /$ día no constituyen un riesgo de aborto espontáneo (41). La mejor aproximación a la correlación entre el consumo de café y el aborto espontáneo, proviene de un estudio en el cual se midió los niveles plasmáticos de paraxantina, un metabolito de la cafeína, con lo cual fue posible obtener una estimación mucho más exacta del consumo de café durante el período de embarazo estudiado, sugiriendo que solo consumos superiores a $600 \mathrm{mg} /$ día de cafeína pueden constituir un mayor riesgo de aborto espontáneo (46). La conclusión de este estudio avala lo ya propuesto, en el sentido que un consumo no superior de $300 \mathrm{mg}$ /día no constituye un factor de riesgo de aborto espontáneo (41).

\section{El café, la cafeína y la diabetes gestacional}

La diabetes gestacional afecta en promedio al $14 \%$ de las embarazadas (47). La patología puede superponerse a una insulino resistencia ya existente antes del embarazo y hacerse crónica, derivando en un daño a las células beta del páncreas (48). Las mujeres con embarazos complicados por la diabetes gestacional tienen un alto riesgo de desarrollar diabetes tipo 2 de por vida (49). Los estudios epidemiológicos indican que el consumo prolongado de café se asocia con un menor riesgo de desarrollo de diabetes tipo 2 en la adultez (50), aunque el consumo agudo puede asociarse con una menor sensibilidad a la insulina (51), lo cual ha motivado a numerosos investigadores a estudiar el efecto del café y de la cafeína durante el embarazo y su relación con la diabetes gestacional. Lo estudios sobre el efecto de la cafeína en la diabetes gestacional son relativamente recientes, ya que el primero de ellos se publicó en 2002 (52) y a partir de este estudio todos los estudios posteriores y meta análisis de cohortes han encontrado una correlación inversa de dosis-respuesta entre el consumo de café y la mayor incidencia de diabetes gestacional (50, 53-55).

\section{La cafeína, el crecimiento fetal $y$ el peso al nacer}

La cafeína atraviesa fácilmente la placenta con lo cual puede alcanzar concentraciones relativamente altas en el líquido amniótico y en la sangre fetal (39). Durante los años 70 algunos estudios sugirieron que el consumo materno de cafeína podría producir reducción del crecimiento fetal, prematurez y bajo peso al nacer, lo cual llevó a la recomendación de disminuir el consumo de café durante el período gestacional (56). Los estudios epidemiológicos que han examinado posteriormente el efecto del consumo de café en el crecimiento fetal y en el peso al nacer, son controversiales en sus resultados, principalmente debido a la inexactitud en la estimación del café consumido por las encuestadas y la presencia de factores confundentes (tabaquismo y consumo de alcohol, entre otros) (57). Estos estudios han considerado retardos del crecimiento fetal menores que el percentil 10 en relación a la edad gestacional y pesos al nacer inferiores a $2.500 \mathrm{~g}$. Un estudio prospectivo que incorporó un gran número de encuestadas, asoció el consumo de cafeína con una pequeña pero significativa disminución del peso al nacer (28 g por cada 100 mg de cafeína consumida al día), sin embargo esta significancia sólo se expresa cuando el consumo de cafeína es superior a $600 \mathrm{mg} /$ día (58). La conclusión general, proveniente del análisis de los estudios realizados a la fecha (1996-2006), es que un consumo no superior a $300 \mathrm{mg}$ /día de cafeína, no afecta el crecimiento fetal y el peso al nacer (59).

\section{La cafeína y defectos al nacimiento}

La administración de cafeína durante la preñez de diferentes especies animales, causa malformaciones congénitas. Sin embargo, estos resultados se obtienen con la administración de altas dosis de cafeína las que generalmente son tóxicas para la propia madre (60). La mayoría de los estudios epidemiológicos no han encontrado asociación entre el consumo de cafeína, o de café, con el riesgo de malformaciones congénitas en el rango de 300-1000 mg/día de cafeína (41). Más recientemente se ha demostrado que el consumo moderado de café $(\geq$ 300 mg cafeína) no constituye un riesgo de malformaciones y hendiduras orofaciales en el feto (61).

\section{Cafeína y lactancia}

La cafeína es detectable en la leche materna 15 minutos después de la ingesta de café, alcanzando un máximo de concentración una hora después del consumo (62). Después del consumo de 335 mg de cafeína la cantidad de cafeína aportada por la leche materna durante un período de 24 horas ha sido estimada en menos de $2 \mathrm{mg}$ (62). Un consumo diario de cafeína de $500 \mathrm{mg}$ 
produce un aporte de cafeína al lactante de 0,3-1,0 mg/ $\mathrm{kg}$ de peso corporal, lo que es considerado irrelevante (63). Aunque se ha informado que un alto consumo de café puede producir irritabilidad y alteraciones del sueño de los bebés, se considera que el consumo moderado de café (dos a tres tazas al día) no tiene efectos adversos en la salud del lactante. La Academia Americana de Pediatría (64) incorpora a la cafeína dentro de los productos que no son incompatibles con la lactancia materna. Una revisión reciente (2009) concluye que si bien la mujer embarazada, la mujer en edad fértil y los niños, constituyen un grupo de riego en relación al consumo de café, un consumo moderado de cafeína $\leq 300 \mathrm{mg} /$ día (equivalente a $4,6 \mathrm{mg} / \mathrm{kg}$ de peso corporal) en los adultos y $\leq 2,5 \mathrm{mg} / \mathrm{kg}$ de peso corporal en los niños, no constituye un riesgo de enfermedad (65).

\section{CONCLUSIONES}

En general, el consumo excesivo de café, o de cafeína, se ha asociado con un mayor riesgo de hipertensión y por consiguiente con un mayor riesgo cardiovascular, aspecto cuya importancia en la actualidad puede ser debatible si se compara con los efectos benéficos de la cafeína que han sido observados más recientemente. En efecto, estos resultados pueden conducir a romper o a modificar "mitos" establecidos sobre el café y la cafeína. El efecto protector del consumo moderado de café en el riesgo de IM, o en la presión arterial, o la no modificación de los niveles plasmáticos de la proteína $\mathrm{C}$ reactiva. La restricción del consumo de café durante el embarazo, también amerita ser revisada. Esto no significa que sea necesario estimular el consumo de café durante este período, más bien, los resultados apuntan a que el consumo moderado de café puede ser permitido, bajo supervisión médica, en aquellas embarazadas que antes del embarazo tenían la costumbre de consumir café. Lo mismo ocurriría en la lactancia. Estos hallazgos apuntan a que aún nos falta mucho por conocer sobre esta tradicional bebida y sobre todo acerca de su componente activo más importante, la cafeína. Posiblemente, futuras investigaciones encontrarán otros efectos beneficiosos del café los que podrán, de alguna manera, compensar o contrarrestar aquellos efectos no benéficos, aunque no del todo demostrados, tradicionalmente asociados al consumo de café y a la cafeína. Hay, hay que considerar los efectos de otras sustancias presentes en el café, como por ejemplo el ácido clorogénico y otros antioxidantes, el alto contenido de magnesio, de potasio, de niacina, entre otros, los que pueden sinergizar los efectos atribuidos a la cafeína (66). El consumo moderado de café, como el de muchas otras sustancias de origen natural, parecer aportar muchos más efectos benéficos que perjudiciales en nuestra salud. Es interesante destacar la opinión de algunos investigadores en el sentido que si el café se hubiese descubierto recientemente, con toda seguridad habría sido calificado como un "alimento funcional" (67). La pregunta obvia sigue siendo ¿es saludable consumir café?, la evidencia clínica y epidemiológica parece indicarlo, siempre y cuando el consumo sea moderado y que no se acompañe de tabaquismo, alcoholismo, sobrepeso, falta de ejercicio, o por una dieta desequilibrada, con abundancia de carbohidratos refinados, grasas saturadas e isómeros trans, estos últimos suficientemente caracterizados como dañinos (68).

\section{RESUMEN}

La cafeína es el principal componente activo del café y los efectos del consumo de café se asocian mayoritariamente a la cafeína, una metilxantina que actúa como antagonista de los receptores de adenina en el sistema nervioso. Son numerosos los efectos en la salud atribuidos a la cafeína, siendo algunos benéficos y otros deletéreos: en la salud cardiovascular, diabetes tipo 2, tolerancia a la glucosa y sensibilidad a la insulina, en la cirrosis hepática y el carcinoma hepatocelular, entre otros efectos. Este artículo realiza una revisión de dos aspectos de preocupación en la salud pública relacionados con el consumo de café y la cafeína: su efecto en la salud cardiovascular y en la salud maternal (período perinatal). La conclusión general es que el consumo de cafeína, hasta $300 \mathrm{mg} /$ día, no constituye un mayor riesgo de infarto al miocardio, de hipertensión, o de modificación de los niveles plasmáticos de indicadores de riesgo cardiovascular, como la proteína $\mathrm{C}$ reactiva y la homocisteína. En relación a la salud maternal, las conclusiones son similares, un consumo moderado de cafeína (300 mg/día o menos) no constituye un riesgo de menor concepción, de diabetes gestacional, de menor crecimiento fetal, o de defectos congénitos. La ausencia de riesgo, sin embargo, no constituye un estímulo al consumo de café durante el embarazo. Si el consumo de café por parte de la futura madre es moderado, puede considerarse la continuación del consumo durante el embarazo bajo supervisión médica.

Palabras clave: Consumo de café, ingestión de cafeína, efectos metabólicos, salud cardiovascular, salud maternal.

Dirigir la correspondencia a:

Profesor

Alfonso Valenzuela B.

Centro de Lípidos

Instituto de Nutrición y Tecnología de los Alimentos (INTA) 
Universidad de Chile

Casilla 138-11

Av. El Líbano 5524 - Macul

Santiago, Chile

Fono: 56-2-9781449

FAX: 56-2-2214030

E-mail: avalenzu@inta.cl

\section{BIBLIOGRAFÍA}

1. Nacional Coffee Association, Coffee drinking trends survey. 2000.

2. Spiller MA. The Chemical components of Coffee. En: Caffeine. Spiller, G. A. Ed. CRC Press, Boca Raton. 1998, pp 97-161.

3. Higdon JV, Frei B. Coffee and Health: A review of recent Human Research. Crit Rev Food Sci Nutr. 2006; 46: 101-23.

4. Lundsberg L. Caffeine consumption. En: Spiller, G. A. Ed. Caffeine, Boca Ratón, New York: CRC Press, 1998, pp 200-5.

5. McCusker RR, Goldenberg BA, Cone EJ. Caffeine content of specialty coffees. J Anal Toxicol. 2003; 27: 520-22.

6. Crews, HM., Olivier L, Wilson LA.. Urinary biomarkers for assessing dietary exposure to caffeine. Food Addit. Contam. 2001; 18:1075-87.

7. Cornelis MC, El-Sohemy A, Kabagambe F K, Campos H. CYP1A2 genotype and risk of myocardial infarction. JAMA 2006; 295: 1135-41).

8. James JE. Critical review of dietary caffeine and blood pressure: A relationship that should be taken more seriously. Psychosom. Med 2004; 66: 63-71.

9. Wardas J. Neuroprotective role of adenosine in the CNS. Pol J Pharmacol. 2002; 54: 313-26.

10. Black AM, Pandya S, Clark D, Armstrong E, Yager J. Effect of caffeine and morphine on the developing pre-mature brain. Brain Res. 2008; 1219: 136-42.

11. Turner CP, Seli M, Steward W, Yan H, Johansson B, Fredholm B, Blackburn M, Rivkees S. A1 adenosine receptors mediate hypoxia-induced ventriculomegaly. Proc Natl Acad Sci U.S. 2003; 100: 11718-22.

12. Tavani A, Bertuzzi M, Gallus S. Risk factors for non fatal acute myocardial infarction in Italian women. Prev Med. 2004; 39: 128-34.

13. Mineharu Y, Koizumi A, Wada Y, Iso H, Watanabe Y. Coffee, green tea, black tea, and oolong tea consumption and risk of mortality from cardiovascular disease in Japanese men and women. J Epidemiol Comm Health. 2009; 23: 123-9.

14. Bonita JS, Mandarano M, Shuta D,Vinson J. Coffee and cardiovascular disease: In Vitro, cellular, animal, and human studies. Pharmacol Res. 2007; 55: 187-98.

15. Greenberg JA, Dunbar C, Schnoll R, Kokolis R, Kokolis S, Kassotis J. Caffeinated beverage intake and the risk of heart disease mortality in the elderly: a prospective analysis. Am J Clin Nutr. 2007; 85: 392-8.

16. Panagiotakos DB, Pitsavos C, Chrysohoou C, Kokkinos P, Toutouzas P, Stefanadis C. The J-shaped effect of coffee consumption on the risk of developing acute coronary syndrome: the CARDIO 2000 case-control study. J Nutr. 2003; 133: 3228-32.

17. De Vreede-Swagemakers JJ, Gorgels AP, Werjemberg MP. Risk indicators for out-of-hospital cardiac arrest in patients with coronary artery disease. J Clin Epidemiol. 1999; 52: 601-7.

18. Mukamal KJ, Maclure M, Muller JE. Caffeinated coffee consumption and mortality after acute myocardial infarction. Am Heart J. 2004; 147: 999-1004.

19. Levy D, Larson MG, Vasan RS, Kannel WB, Ho KK. The progression from hypertension to congestive heart failure. JAMA 1996; 275: 1557-62.

20. Lane JD, Adcock RA, Williams RB, Kuhn CM. Caffeine effects on cardiovascular and neuroendocrine responses to acute psychosocial stress and their relationship to level of habitual caffeine consumption. Psychosom Med. 1990; 52: 320-36.

21. Winkelmayer WC, Stampfer MJ, Willett W, Curhan G. Habitual caffeine intake and the risk of hypertension in women. JAMA 2005; 294: 2330-5.

22. Hamer M. Coffee and health: explaining conflicting results in hypertension. J Hum Hypertens. 2006; 20: 909-12.

23. Vinson JA. Caffeine and incident hypertension in women. JAMA 2006; 295: 2135-9.

24. Frost L, Vestergaard P. Caffeine and risk of atrial fibrillation or flutter: the Danish Diet, Cancer, and Health Study. Am J Clin Nutr. 2005; 81: 578-82.

25. López-García E., van Dam R., Willett W, Rimm E, Manson J, Stampfer M, Rexrode K, Hu F. Coffee consumption and coronary heart disease in men and women: a prospective cohort study. Circulation 2006; 113: 2045-53.

26. Husemoen L, Thomsen T, Fenger M, Jorgensen T. Effect of lifestyle factors on plasma total homocysteine concentrations in relation to MTHFR(C677T) genotype. Eur J Clin Nutr. 2004; 58: 1142-50.

27. Urgert R., van Vliet, T, Zock P, Katan M.. Heavy coffee consumption and plasma homocysteine: A randomized controlled trial in healthy volunteers. Am J Clin Nutr. 2000; 72: 1107-10.

28. Strandhagen E, Zetterberg H, Aires N. The methy- 
lenetetrahydrofolate reductase C677T polymorphism is a major determinant of coffee-induced increase of plasma homocysteine. A randomized placebo controlled study. Int J Mol Med. 2004; 13: 811-5.

29. Strandhagen E, Landaas S, Thelle D. Folic acid supplement decreases the homocysteine increasing effect of filtered coffee. A randomized placebocontrolled study. Eur J Clin Nutr. 2003; 57: 1411-7.

30. López-García E, van Dam RM, Li T, RodríguezArtalejo F, Hu F. The relationship of coffee consumption with mortality. Ann Int Med. 2008; 148: 904-14.

31. López-García E, van Dam RM, Qi L, Hu F. Coffee consumption and markers of inflammation and endothelial dysfunction in healthy and diabetic women. Am J Clin Nutr. 266; 84: 888-93.

32. Balk, L., Hoekstra, T., Twisk, J. Relationship between long-term coffee consumption and components of metabolic syndrome: The Amsterdam Growth and Health Longitudinal Study. Eur J Epidemiol. 2009; 24: 203-9.

33. Kirkinen P, Jouppila P, Koivula A, Vuori J, Puukka $M$. The effect of caffeine on placental and fetal blood flow in human pregnancy. Am J Obstet Gynecol. 1983; 147: 939-42.

34. Kalow W, Tang BK. Use of caffeine metabolite ratios to explore CYP1A2 and xanthine oxidase activities. Clin Pharmacol Ther. 1991; 50: 508-19.

35. Santos I, Victora C, Huttly S, Carvalhal J. Caffeine intake and low birth weight: A population-based case-control study. Am J Epidemiol. 1998; 147: 620-7.

36. Ginsberg G, Hattis D, Russ A, Sonawane B. Physiologicaly based pharmacokinetic (PBPK) modelling of caffeine and theophylline in neonates and adults: Implications for assessing children's risk from environmental agents. J Toxicol Environ. Health 2004; 67: 297-329.

37. Aranda J, Louridas A, Vitullo B, Thom P, Aldridge A, Haber R. Metabolism of theophyline to caffeine in human fetal liver. Science 1979; 206: 1319- 21.

38. Liu YA, Ostlund E, Fried G. Endothelium-induced contractions in human placental blood vessels are enhanced in intra uterine growth retardation, and modulated by agents that regulate levels of intracellular calcium. Acta Physiol Scand. 1995; 155: 405-14.

39. Grosso L, Bracken MB. Caffeine metabolism, genetics, and perinatal outcomes: a review of exposure assessment considerations during pregnancy. Ann Epidemiol. 2005; 15; 460-6.
40. U.S. Department of Agriculture, Food Surveys Research Group. 1994-1996 Continuing Survey of Food Intakes by Individuals: Table set 10 . Available at: http//www.brac.usda.gov/foodsurvey/home. htm., 2005.

41. Nawrot P, Jordan S, Eastwood J. Effects of caffeine on human health. Food Addit Contam. 2003; 20: 1-30.

42. Alderete E, Eskenazi B, Sholtz R. Effect of cigarette smoking and coffee drinking on time to conception. Epidemiology, 1995; 6: 403-8.

43. Caan B, Quesenberry CP, Coates AO. Differences in fertility associated with caffeinated beverage consumption. Am J Public Health. 1998; 88: 270-4.

44. Leviton A, Cowan L. A review of the literature relating caffeine consumption by women to their risk of reproductive hazards. Food Chem Toxicol. 2002; 40: 1271-310.

45. Signorello LB, McLaughlin JK. Maternal caffeine consumption and spontaneous abortion: a review of the epidemiologic evidence. Epidemiol. 2004; 15: 229-39.

46. Klebanoff MA, Levine RJ, Dersimonian R. Maternal serum paraxanthine, a caffeine metabolite, and the risk of spontaneous abortion. N Engl J Med. 1999; 341: 1639-44.

47. American Diabetes Association. Diagnosis and classification of diabetes mellitus. Diabetes Care. 2005; 28 (suppl 1): S37-S42.

48. Buchanan TA, Xiang AH. Gestational diabetes mellitus. J Clin Invest. 2005; 115: 485-91.

49. Kim C, Newton KM, Knopp RH. Gestational diabetes and the incidence of type 2 diabetes. A systematic review. Diabetes Care. 2002; 25: 1862-8.

50. van Dam R M, Hu FB. Coffee consumption and risk of type 2 diabetes: a systematic review. JAMA 2005; 294: 97-104.

51. Keijzers GB, De Galan BE, Tack CH, Smits P. Caffeine can decrease insulin sensitivity in humans. Diabetes Care. 2002; 25: 364-9.

52. van Dam RM, Feskens EJ. Coffee consumption and risk of type 2 diabetes mellitus. Lancet. 2002; 360: 1477-8.

53. Reunanen A, Heliovaara M, Aho K. Coffee consumption and risk of type 2 diabetes mellitus. Lancet. 2003; 360: 1477-8.

54. Tuomilehto J, Hu G, Bidel S. Coffee consumption and the risk of type 2 diabetes mellitus among middle-aged Finnish men and women. JAMA 2004; 291: 1213-19.

55. Adeney K, Williams M, Schiff M, Qiu C, Sorensen T. Coffee consumption and the risk of gestational 
diabetes mellitus. Acta Obstetr Gynecol. 2007; 86: 161-6.

56. Watkinson B, Fried PA. Maternal caffeine use before, during and after pregnancy and effects upon offpring. Neurobehav. Toxicol Teratol. 1985; 7 : 9-17.

57. Martin TR, Bracken MB. The association between low birth weight and caffeine consumption during pregnancy. Am J Epidemiol. 1987; 126: 813-21.

58. Bracken MB, Triche EW, Belanger K. Association of maternal caffeine consumption with decrements in fetal growth. Am J Epidemiol. 2003; 157: 456-66.

59. De Resende AH, Silva N, Santos I, Kac G. Caffeine consumption during pregnancy and prevalence of low birth weight and prematurity: a systematic review. Cad Saude Pub Río de Janeiro. 2007; 23: 2807-19.

60. Christian MS, Brent RL. Teratogen update: evaluation of the reproductive and developmental risks of caffeine. Teratology. 2001; 64: 51-78.

61. Marte A, Allen J, Rolv W, Lene L, Drevon C. Maternal consumption of coffee and caffeine-containing beverages and oral clefts: A population-based casecontrol study in Norway. Am J Epidemiol. 2009;
169: 1216-22.

62. Berlin CM, Denson HM, Daniel CH, Ward RM. Disposition of dietary caffeine in milk, saliva, and plasma of lactating women. Pediatrics. 1984; 73: 59-63.

63. Ryu JE. Caffeine in human milk and in serum of breast-fed infants. Dev Pharmacol Ther. 1985; 8: 329-37.

64. American Academy of Pediatrics Committee on Drugs. Transfer of drugs and other chemicals into human milk. Pediatrics. 2001; 108: 776-89.

65. Kuzkowski, K. Caffeine in pregnancy. Arch Gynecol Obstet. 2009; 280: 695-8.

66. Ranhein T, Halvorsen B. Coffee consumption and human health - beneficial or detrimental? Mechanisms for effects of coffee consumption on different risk factors for cardiovascular disease and type 2 diabetes mellitus. Mol Nutr Food Res. 2005; 49; 274-84.

67. Katan M, Schouten E. Caffeine and arrhythmia. Am J Clin Nutr. 2005; 81: 539-40.

68. Valenzuela A. Acidos grasos con isomería trans I. Su origen y los efectos en la salud. Rev Chil Nutr. 2008; 35: 162-71 